\title{
Right ventricular papillary muscle approximation as a novel technique of valve repair for functional tricuspid regurgitation in an ex vivo porcine model
}

\author{
Haruo Yamauchi, MD, PhD, ${ }^{\mathrm{a}}$ Nikolay V. Vasilyev, MD, ${ }^{\mathrm{a}}$ Gerald R. Marx, MD, ${ }^{\mathrm{b}}$ Hugo Loyola, MS, ${ }^{\mathrm{a}}$ \\ Muralidhar Padala, $\mathrm{PhD},{ }^{\mathrm{c}}$ Ajit P. Yoganathan, $\mathrm{PhD},{ }^{\mathrm{d}}$ and Pedro J. del Nido, $\mathrm{MD}^{\mathrm{a}}$
}

\begin{abstract}
Objectives: Annuloplasty for functional tricuspid regurgitation may sometimes be ineffective because of chamber dilation and valve tethering. This study compared a novel technique, right ventricle (RV)-papillary muscle approximation, with annuloplasty in experimentally-produced tricuspid regurgitation.
\end{abstract}

\begin{abstract}
Methods: RVs of isolated porcine hearts $(\mathrm{n}=10)$ were statically pressurized, which led to RV dilation and central tricuspid regurgitation. Regurgitant flow was measured with a saline solution-filled column. The head of the anterior papillary muscle was approximated to 4 points on the ventricular septum. Next, a prosthetic ring was implanted, and then RV-papillary muscle approximation was combined. Tricuspid annular dimension, RV geometry, and tricuspid valve tethering were analyzed with 3-dimensional echocardiography.

Results: Tricuspid regurgitation $(2270 \pm 186 \mathrm{~mL} / \mathrm{min})$ was reduced by RV-papillary muscle approximation alone $(214 \pm 45 \mathrm{~mL} / \mathrm{min} ; P<.05)$ more than by annuloplasty alone $(724 \pm 166 \mathrm{~mL} / \mathrm{min} ; P<.05)$. Combined $\mathrm{RV}$-papillary muscle approximation and annuloplasty resulted in the least regurgitation $(80 \pm 39 \mathrm{~mL} / \mathrm{min})$. $\mathrm{RV}$-papillary muscle approximation reduced tricuspid septolateral diameter $(25 \% ; P<.05)$, and annular area $(23 \% ; P<.05)$, as did annuloplasty. RV-papillary muscle approximation also reduced RV sphericity index $(33 \% ; P<.05)$ and tricuspid tethering height $(54 \% ; P<.05)$, whereas annuloplasty did not. Direction of $\mathrm{RV}$-papillary muscle approximation did not independently affect outcomes.
\end{abstract}

Conclusions: This ex vivo study suggests that RV-papillary muscle approximation potentially repairs tricuspid regurgitation better than annuloplasty by improving ventricular sphericity and valve tethering as well as annular dimension. (J Thorac Cardiovasc Surg 2012;144:235-42)

Functional tricuspid regurgitation (TR) is commonly caused by tricuspid annular dilation and right ventricular enlargement, which is often secondary to left-sided valve and ventricular dysfunction or to pulmonary hypertension and adversely affects cardiac-related mortality. ${ }^{1,2}$ Tricuspid annuloplasty (TAP) for functional TR, concomitant with repair of left-sided valve disease, generally results in good outcome; still, no fewer than $10 \%$ of the patients have residual or recurrent moderate to severe TR on follow-up. ${ }^{3}$ Tricuspid valve tethering, presumably as a result of outward displacement of papillary muscles (PMs) in the dilated right ventricle from pulmonary hypertension, has been reported

\footnotetext{
From the Departments of Cardiac Surgery ${ }^{\mathrm{a}}$ and Cardiology, ${ }^{\mathrm{b}}$ Children's Hospital Boston, Harvard Medical School, Boston, Mass; the Division of Cardiothoracic Surgery, ${ }^{\mathrm{c}}$ Emory University School of Medicine, Atlanta, Ga; and The Wallace H. Coulter Department of Biomedical Engineering, ${ }^{\mathrm{d}}$ Georgia Institute of Technology/Emory University, Atlanta, Ga.

Supported in part by National Institute of Health grants HL-73647 and HL-089269 (PJdN).

Disclosures: Authors have nothing to disclose with regard to commercial support.

Received for publication Oct 19, 2011; revisions received Dec 30, 2011; accepted for publication Jan 6, 2012; available ahead of print Feb 20, 2012.

Address for reprints: Pedro J. del Nido, MD, 300 Longwood Ave, Boston, MA 02115

(E-mail: Pedro.delnido@childrens.harvard.edu).

0022-5223/\$36.00

Copyright (c) 2012 by The American Association for Thoracic Surgery

doi:10.1016/j.jtcvs.2012.01.028
}

as a factor that decreases the durability of TAP. ${ }^{4-6}$ This observation is supported by the recent in vitro study revealing that tricuspid annular dilation and $\mathrm{PM}$ displacement independently cause TR. ${ }^{7}$ A treatment strategy that addresses both PM displacement and annular dilation is therefore likely to be more effective than TAP alone.

In functional mitral regurgitation, valve tethering from PM displacement is also considered an important mechanism. ${ }^{89}$ Recent reports describing novel intraventricular techniques that relocate the displaced posterior PM to its normal position ${ }^{10,11}$ or reposition both PMs to their midline $^{12-15}$ have shown successful treatment of functional mitral regurgitation with reduction of mitral valve tethering. ${ }^{10,12,14}$ Inspired by these observations, we developed an analogous technique of right ventricle-PM approximation (RV-PMA) to treat functional TR at the level of the PMs. This involves approximating the head of the anterior PM arising from the right ventricular free wall to the ventricular septum. The objective of this study was to examine the effects of the RV-PMA technique on TR, tricuspid annular dimension, right ventricular geometry, and tricuspid valve tethering and to compare them with TAP in an ex vivo TR model with isolated porcine hearts analyzed with 3-dimensional (3D) echocardiography. 


\section{Abbreviations and Acronyms \\ 3D $=3$-dimensional \\ A-M = anterior papillary muscle to medial papillary muscle [suture position] \\ A-P $=$ anterior papillary muscle to posterior papillary muscle [suture position] \\ A-S1 = anterior papillary muscle to the midpoint between medial and posterior papillary muscles [suture position] \\ A-S2 = anterior papillary muscle to intersection of septum and the perpendicular line on the suture of anterior papillary muscle [suture position] \\ $\mathrm{PM}=$ papillary muscle \\ RV- $=$ right ventricle-papillary muscle \\ PMA approximation \\ TAP $=$ tricuspid annuloplasty \\ TR = tricuspid regurgitation}

\section{MATERIALS AND METHODS \\ Ex Vivo Isolated Heart Model}

Isolated fresh porcine hearts were obtained from a slaughterhouse. Hearts were selected on the basis of the intact state of the right ventricles. Approximately $30 \%$ of these hearts were selected for the experiments by excluding the hearts with injuries of tricuspid valve, right ventricular wall, or ventricular septum. The hearts were then tested by manual saline solution injection. Hearts that had tricuspid valve leaflet prolapse or were without apparent TR were also excluded. The hearts $(n=10 ; 424 \pm 14 \mathrm{~g})$ that showed easily visible central TR were used for this ex vivo model. The posterior wall of the left ventricle was resected, and the remainder of the heart was mounted on a flow loop in a saline solution-filled tank $\left(37^{\circ} \mathrm{C}\right)$. Pulmonary valve leaflets were removed, and the pulmonary artery was connected to saline solution-filled tubing and column. The top of the saline solution-filled column was open to the atmosphere. The height of the saline solution-filled column was varied among 27, 55, and $82 \mathrm{~cm}$ above the surface in the tank to pressurize the right ventricle to 20,40 , and 60 $\mathrm{mm} \mathrm{Hg}$, respectively. The closing volume of the tricuspid valve was excluded from the TR flow by starting measurements after the tricuspid valve was completely closed under the pressure. Then the TR flow (in milliliters per minute) was calculated by measuring the reduced volume in the column from 5 to 60 seconds, depending on the amount of TR flow (Figure 1, A). The actual changed heights of the saline solution-filled column were at most $1.2,2.0$, and $2.9 \mathrm{~cm}$, equivalent to $0.9,1.5$, and $2.1 \mathrm{~mm} \mathrm{Hg}$, below the set height under 20, 40, and $60 \mathrm{~mm} \mathrm{Hg}$ pressures, respectively.

\section{Surgical Technique of RV-PMA}

Sutures (2-0 Ethibond; Ethicon, Inc, Somerville, NJ) were placed with a polytetrafluoroethylene pledget $(7 \mathrm{~mm}$ in diameter) at the head of anterior PM and pulled toward 4 points on the ventricular septum: medial PM (A-M), posterior PM (A-P), the midpoint between medial and posterior PMs (A-S1), and the intersection of the septum and the perpendicular line on the suture of anterior PM (A-S2). The virtual plane in which the sutures were pulled and the plane of tricuspid annulus were kept parallel (Figure 1, $B$ and $C$ ). The distances between the anterior PM and the septum were reduced by RV-PMA, the extent of which was either $0 \%$ (initial position), $25 \%, 50 \%, 75 \%$, or $100 \%$ (direct contact between the PM and the septum; Figure 1, D). To evaluate annuloplasty, a moderately undersized

prosthetic rigid ring (distance between anteroseptal and posteroseptal commissures $29 \mathrm{~mm}, \mathrm{n}=9 ; 28 \mathrm{~mm}, \mathrm{n}=1$ ), manufactured for research purpose, was sutured onto the tricuspid annulus with 7 to 9 single sutures (3-0 Ethibond; Ethicon) as TAP. The efficacy of this ring in reducing TR has been established in acute and long-term animal models of TR induced by isolated tricuspid annular dilatation. ${ }^{16}$ To evaluate the additive effects of RV-PMA with annuloplasty, the distances between the anterior PM and the septum were reduced again from $0 \%$ to $100 \%$ extent in the hearts after TAP.

\section{Echocardiographic Measurement}

The 3D echocardiographic data were acquired from the hearts under 40 $\mathrm{mm} \mathrm{Hg}$ of right ventricular pressure by the full-volume acquisition mode with a matrix array probe X7-2 (2-7 MHz) on an iE33 system (Philips Healthcare, Andover, Mass). The 3D echocardiographic data were then analyzed offline in the multiplanar 3D quantification mode of Philips Qlab software $^{17}$ (version 8.1 ) to measure the septolateral diameter, the anteroposterior diameter, and the area of the tricuspid annulus (Figure 1,E); the right ventricular sphericity index (RVSI); and the tricuspid valve tethering height (Figure 1, F). For the tricuspid annular area, the most appropriate plane was defined to project some portions of tricuspid annulus as an approximate 2-dimensional shape, because the Qlab acquisition software does not accommodate the direct calculation of the 3D-shaped area of the tricuspid valve. For the RVSI calculation with 3D echocardiographic data, the tricuspid annular line, equivalent to the line for the septolateral diameter, right ventricular long-axis line from the true right ventricular apex to the midpoint of the tricuspid annular line, and the right ventricular shortaxis line between the right ventricular wall and the septum perpendicular to the right ventricular long-axis line at its midpoint were determined. The RVSI was calculated as a ratio of the right ventricular short-axis line to the right ventricular long-axis line (Figure 1,F). For the tricuspid valve tethering height, the largest height between the tricuspid annular line and the coapting point between the tricuspid valve leaflets was determined (Figure $1, F$ ). In addition, acquired 3D data files were exported into the 4-D Echo View software (TomTec, Munich, Germany) to calculate the right ventricular volume by the method of disc summation, as described previously elsewhere. ${ }^{18}$

\section{Statistical Analysis}

Measurements are presented as mean \pm SEM. Statistical significance was evaluated with STATA/IC software (version 10.1; StataCorp LP, College Station, Tex) by repeated measures analysis of variance, followed by comparisons between control and experimental conditions by the Dunnett test or Fisher least significant difference comparison test among multiple groups.

\section{RESULTS \\ Effects of RV-PMA and TAP on Tricuspid Regurgitation Flow}

The effects of RV-PMA on TR were compared with those of TAP in our unique ex vivo porcine heart model. The TR flow decreased proportionately to the extent of RV-PMA (Figure 2, A). Ring TAP alone also reduced the TR flow; however, TAP alone was less effective than $100 \%$ RV-PMA alone (Figure 2, $A$ ). Even with TAP, RV-PMA further reduced TR flow (Figure 2, A). No significant differences were observed among the 4 directions of RV-PMA regardless of TAP (Figure 2, $B$ ). The TR flow changed according to the right ventricular pressure; however, the effects of RV-PMA and TAP persisted under the different right ventricular pressures (Figure 2, C). 


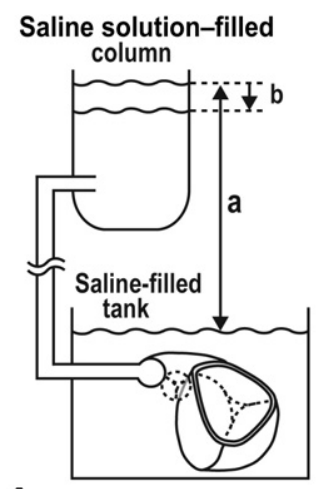

A

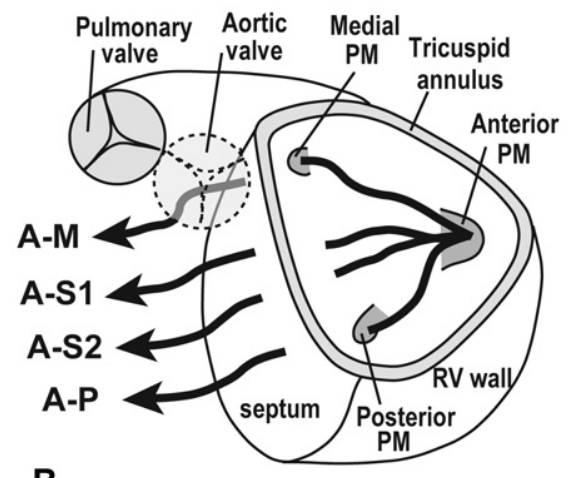

B

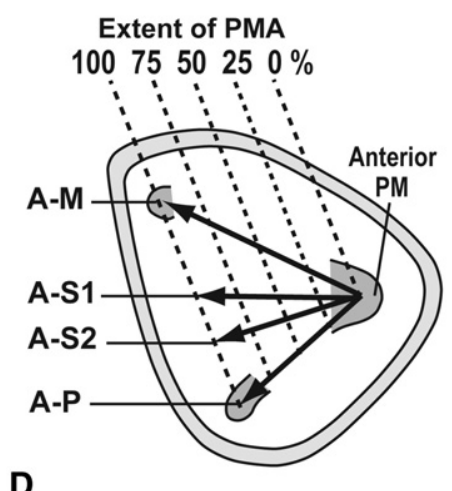

E

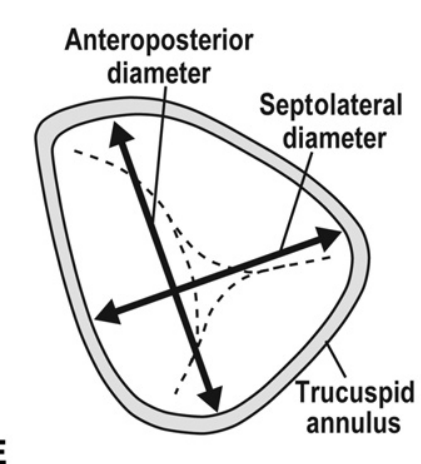

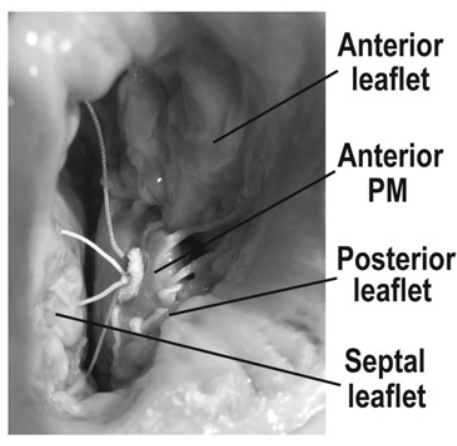

C

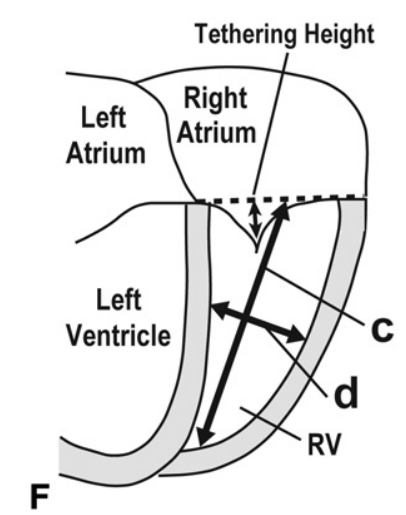

FIGURE 1. Experimental procedure and echocardiographic analysis. A, Experimental setups of saline solution-filled tank and column. Height of the saline solution-filled column above the tank (a) was variable to pressurize the right ventricle $(R V)$ from 20 to $60 \mathrm{~mm} \mathrm{Hg}$. The tricuspid regurgitation flow (in milliliters per minute) was calculated by measuring the reduced height $(b)$ in the column for 5 to 60 seconds after the right ventricle was pressurized and tricuspid valve was closed. B, Schematic drawing of right ventricle-papillary muscle $(P M)$ approximation. The anterior papillary muscle arises from the right ventricular wall. Medial and posterior papillary muscles arise from the ventricular septum. The head of the anterior papillary muscle is approximated to the 4 points of the septum: the medial papillary muscle $(A-M)$, the posterior papillary muscle $(A-P)$, the midpoint between the medial papillary muscle and the posterior papillary muscle $(A-S 1)$, and the intersection of the septum and the perpendicular line on the anterior papillary muscle (A-S2). C, A representative photograph of the tricuspid valve and sutures on the anterior papillary muscle. D, The distance between the anterior papillary muscle and septum is reduced by papillary muscle approximation $(P M A)$ in each direction. $\mathrm{E}$ and $\mathrm{F}$, Three-dimensional echocardiography was used to measure tricuspid annular dimension $(\mathrm{E})$ and right ventricular geometry $(\mathrm{F})$. The right ventricular sphericity index was calculated as a ratio of the right ventricular short-axis line $(d)$ to the right ventricular long-axis line $(c)$.

\section{Effects of RV-PMA and TAP on the Tricuspid Annular Dimension}

The tricuspid annulus was initially distended, and the regurgitant orifice was clearly observed at the center of tricuspid valve leaflets in the $0 \%$ RV-PMA group, whereas the annulus visibly decreased in size and the regurgitant orifice was hardly seen after RV-PMA. The tricuspid annulus, however, became distorted in the A-M group. The location of coapting portions of the 3 leaflets changed from anterior in the A-M group to posterior in the A-P group (Figure 3, A). In 3D echocardiographic analysis, the septolateral diameter of the tricuspid annulus was reduced by RV-PMA alone similar, to the change seen with TAP alone (Figure 3, B). On the other hand, the anteroposterior diameter was not significantly changed by either RV-PMA or TAP (Figure 3, $C$ ). The tricuspid annular area was reduced by RV-PMA alone to the same level as that achieved by TAP alone (Figure 3,
$D$ ). There were no significant differences among the RV-PMA directions in all the parameters (Figure 3, E-G), except that septolateral diameters were smaller in the A-M and A-S1 groups than those in the A-S2 and A-P groups for 50\% RV-PMA (Figure 3,E).

\section{Effects of RV-PMA and TAP on Right Ventricular Geometry and Tricuspid Valve Tethering}

In 3D echocardiographic analysis, both the base of the anterior PM and the lateral portion of the tricuspid annulus were pulled toward the septum by RV-PMA, whereas only the annulus was reduced in size by TAP (Figure $4, A$ ). The RVSI was reduced by RV-PMA alone but was not significantly reduced by TAP alone. Even with TAP, RV-PMA further reduced RVSI (Figure 4, $B$ ). The effects of RV-PMA on the right ventricular volume were analyzed with only the data after TAP, because the large TR flow $(>2000 \mathrm{~mL} / \mathrm{min})$ 

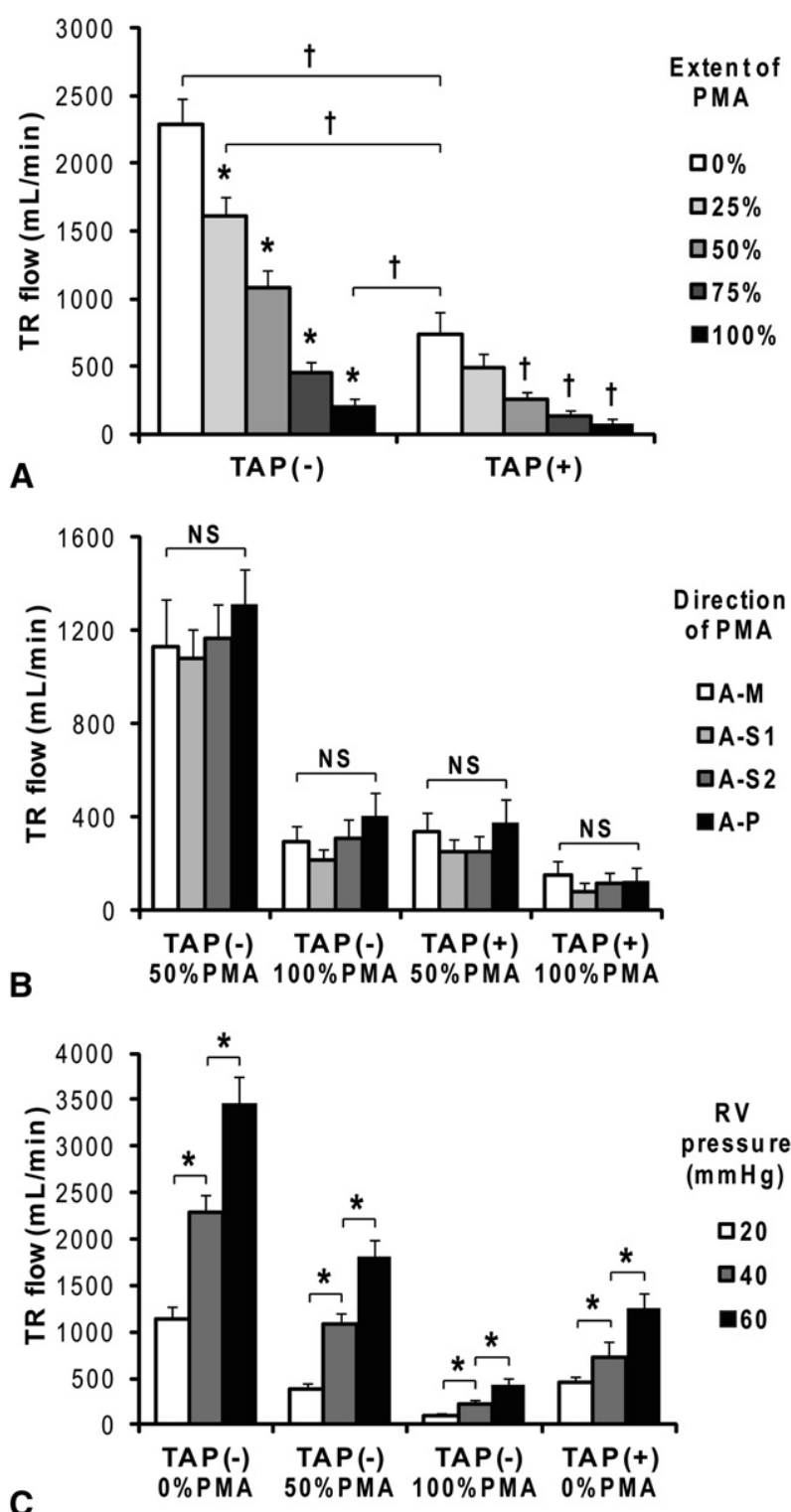

FIGURE 2. Changes of tricuspid regurgitation (TR) flow by right ventricle $(R V)$-papillary muscle approximation (PMA) and tricuspid annuloplasty (TAP). A, Without (-) tricuspid annuloplasty, tricuspid regurgitation flow under $40 \mathrm{~mm} \mathrm{Hg}$ of right ventricular pressure was reduced by right ventricle-papillary muscle approximation. Tricuspid regurgitation flow was also reduced by tricuspid annuloplasty. Even with $(+)$ tricuspid annuloplasty, right ventricle-papillary muscle approximation further reduced tricuspid regurgitation flow. Asterisk indicates $P<.05$ versus no annuloplasty with $0 \%$ papillary muscle approximation by Dunnett $t$ test. Dagger indicates $P<.05$ versus tricuspid annuloplasty with $0 \%$ papillary muscle approximation by Dunnett $t$ test. B, Tricuspid regurgitation flow was not different among the directions of right ventricle-papillary muscle approximation (details of directions given in Figure 1) according to analysis of variance. NS, Not significant. C, Tricuspid regurgitation flow under 20 or $60 \mathrm{~mm} \mathrm{Hg}$ of right ventricular pressure was less or more than that under $40 \mathrm{~mm} \mathrm{Hg}$, respectively, but the effects of right ventricle-papillary muscle approximation and tricuspid annuloplasty were similar regardless of the right ventricular pressure. Asterisk indicates $P<.05$ by least significant difference comparison test. could cause underestimation of the right ventricular volume $(165 \pm 10 \mathrm{~mL})$ in the initial condition of the hearts without RV-PMA or TAP, as compared with the volumes after $50 \%$ RV-PMA $(172 \pm 12 \mathrm{~mL})$ and TAP alone $(184 \pm 12 \mathrm{~mL})$. After TAP, $100 \%$ RV-PMA significantly reduced right ventricular volume (Figure 4,C). The tricuspid valve tethering height was also reduced by RV-PMA alone but not by TAP alone. Even with TAP, RV-PMA reduced tethering height (Figure 4, D). Among the RV-PMA directions, both RVSI and right ventricular volume were less in the A-M group than in the A-P and A-S2 groups with $100 \%$ RV-PMA, whereas a significant difference was shown only in the RVSI with 50\% RV-PMA (Figure 4, $E$ and $F$ ). There were no significant differences among the RV-PMA directions in the analyses of tricuspid valve tethering height (Figure 4, $G$ ).

\section{DISCUSSION}

The results of this ex vivo study investigating the effects of RV-PMA on TR demonstrated several findings. First, RVPMA reduced TR, independent of TAP. Second, RV-PMA reduced the septolateral diameter and the area of tricuspid annulus. Third, RV-PMA reduced the RVSI and the volume of the right ventricle. Fourth, RV-PMA reduced the tricuspid valve tethering height. Finally, TAP reduced the annular dimension but not RVSI or tricuspid valve tethering. These results suggest that RV-PMA potentially reduces TR by addressing right ventricular and tricuspid valve geometry simultaneously.

Although the concept of RV-PMA derives from the experiences with relevant techniques in functional mitral regurgitation, ${ }^{10-15}$ the more complicated PM configuration in the right ventricle makes RV-PM approximation more challenging than the analogous left ventricular operation. As opposed to the prominent dual PM configuration of the normal left ventricle, the right ventricle has asymmetric alignment of the PMs, comprising a large anterior PM on the right ventricular free wall and relatively small medial and posterior PMs on the septal side (Figure 1, $B$ ). The anterior PM is located beneath the commissure of the anterior and posterior leaflets, and it has a number of chordal insertions to both leaflets. The medial and posterior PMs are located beneath the anteroseptal and posteroseptal commissures, respectively, and they have chordal insertions from the corresponding leaflets. The septal leaflet also has multiple chordal attachments directly to the right ventricular septal wall. ${ }^{19}$ With functional TR, the tricuspid annulus dilates by more than $40 \%$, mainly at the anterior and posterior segments. ${ }^{7}$ The septolateral diameter of the annulus is increased depending on the severity of functional TR. ${ }^{20}$ The right ventricular wall concurrently dilates laterally, the sphericity increases, and the anterior PM is displaced laterally, resulting in tricuspid valve tethering and incomplete 


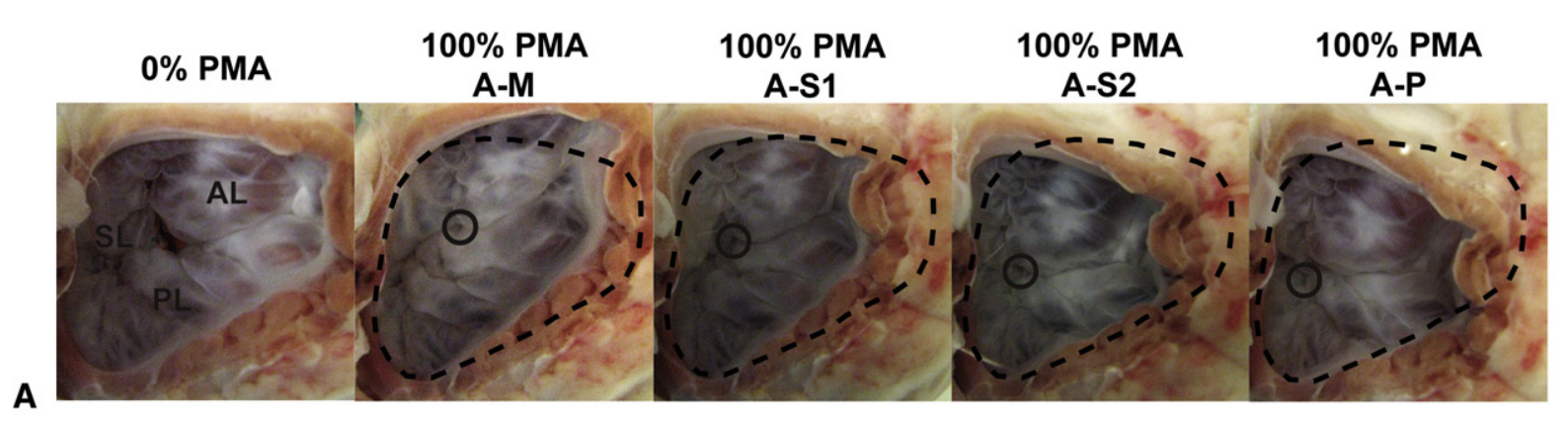

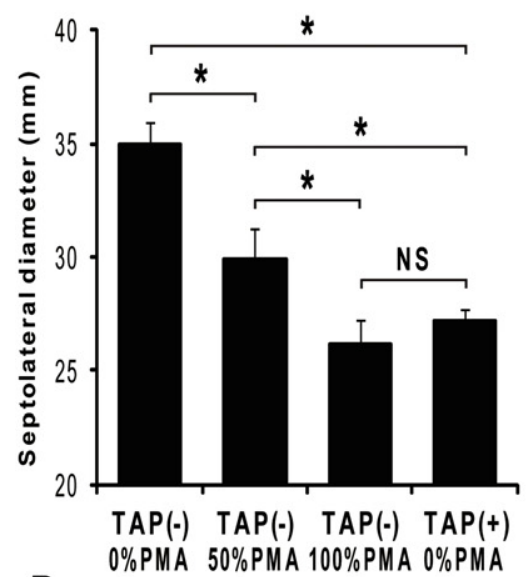

B

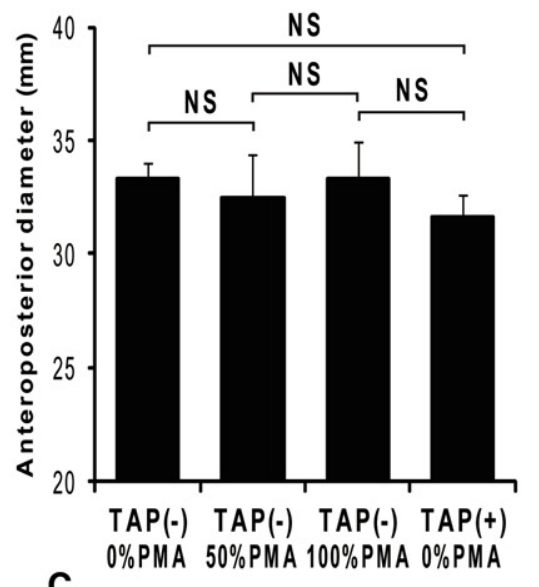

QA.M QA.S1 QA.S2 QA.P

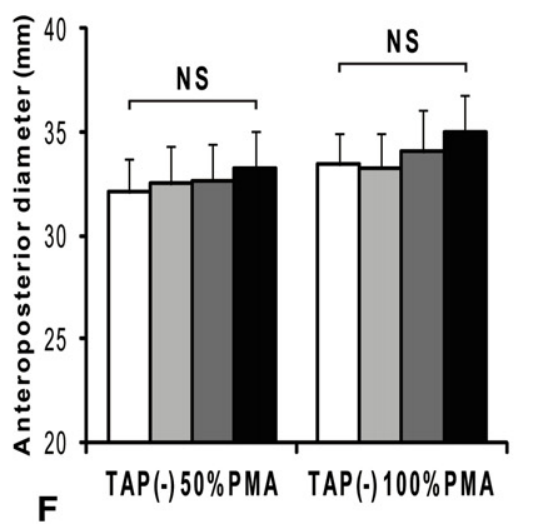

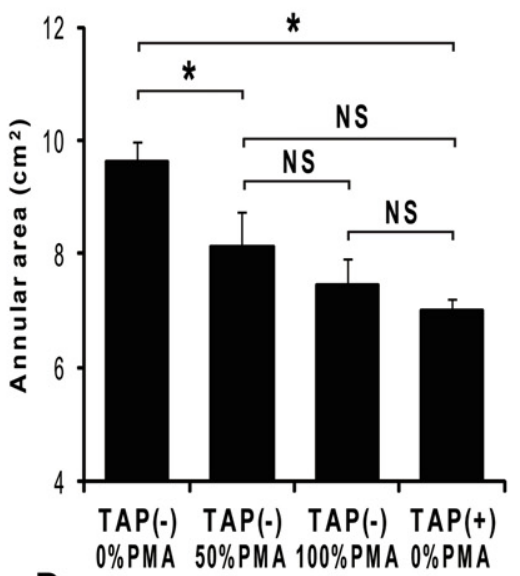

QA.M QA.S1 QA.S2 QA.P

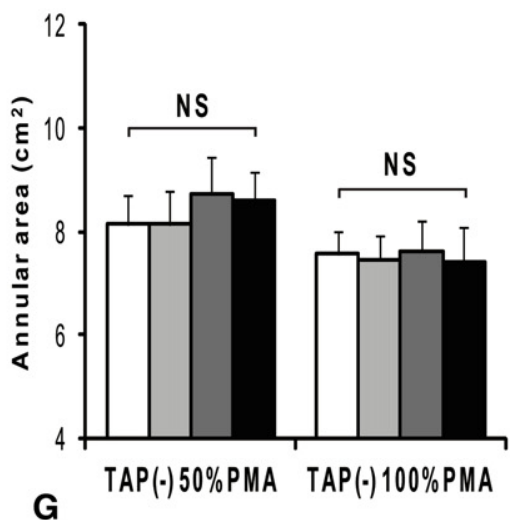

FIGURE 3. Changes in tricuspid annular dimension produced by right ventricle-papillary muscle approximation $(P M A)$ and tricuspid annuloplasty $(T A P)$. A, These are representative photographs of tricuspid valve under $40 \mathrm{~mm} \mathrm{Hg}$ pressure without (-) tricuspid annuloplasty before $(0 \%)$ and after $(100 \%)$ papillary muscle approximation toward the 4 directions (details of directions given in Figure 1). Black dotted lines reflect the contour of the tricuspid annulus before papillary muscle approximation. Black circles indicate coapting portions of 3 leaflets. $A L$, Anterior leaflet; $S L$, septal leaflet; $P L$, posterior leaflet. B through G, According to the 3-dimensional echocardiographic analysis, the septolateral diameter (B) and the area (D) of the tricuspid annulus were reduced by $50 \%$ and $100 \%$ papillary muscle approximation toward the septum (A-S1) as well as by performance of (+) tricuspid annuloplasty, although the anteroposterior diameter of the tricuspid annulus (C) was not changed by either papillary muscle approximation or tricuspid annuloplasty (analysis of variance). Among the directions of papillary muscle approximation, the tricuspid annular diameter (E and F) and area $(\mathrm{G})$ did not differ, except for the septolateral diameter with $50 \%$ papillary muscle approximation, which was less in the A-M and the A-S1 groups than in the A-S2 and the A-P groups (E). Asterisk indicates $P<.05$ by least significant difference comparison test. NS, Not significant.

valve closure. ${ }^{4-6}$ To reverse these pathologic changes, we considered approximation of the anterior PM to the ventricular septum as a reasonable way to reduce the septolateral dimension of the right ventricle. To determine the optimal method of RV-PMA, we investigated the directions and the extent of approximation. 


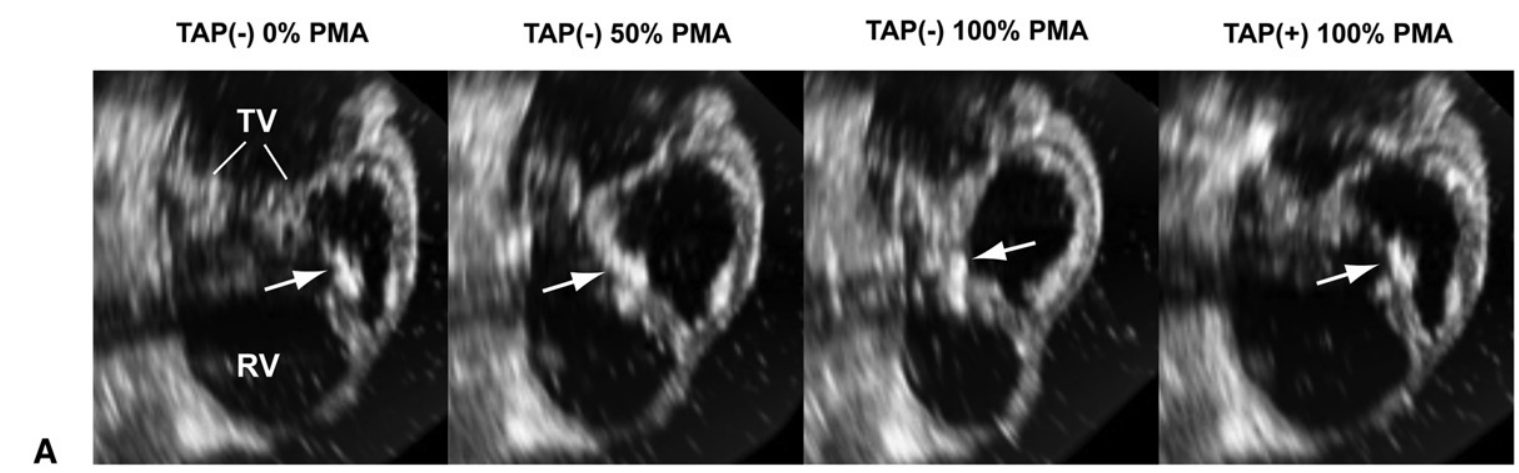

A

口TAP(-) घTAP(+)

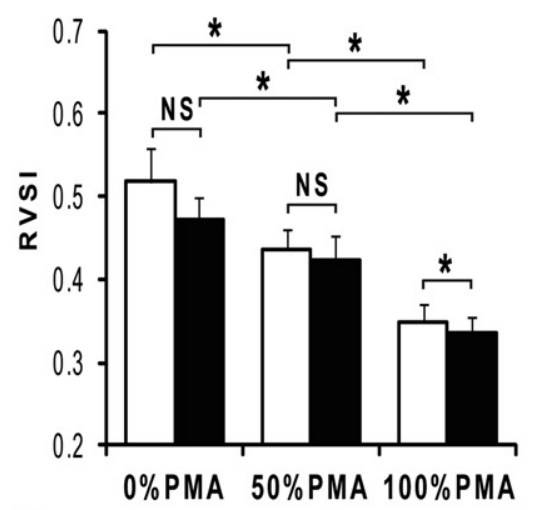

B

QA.M QA.S1 QA.S2 ШA.P

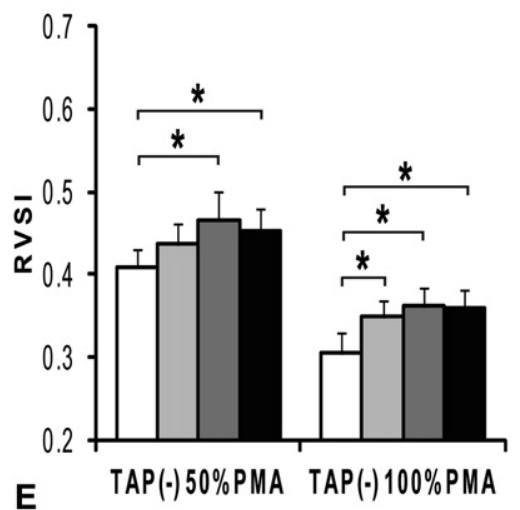

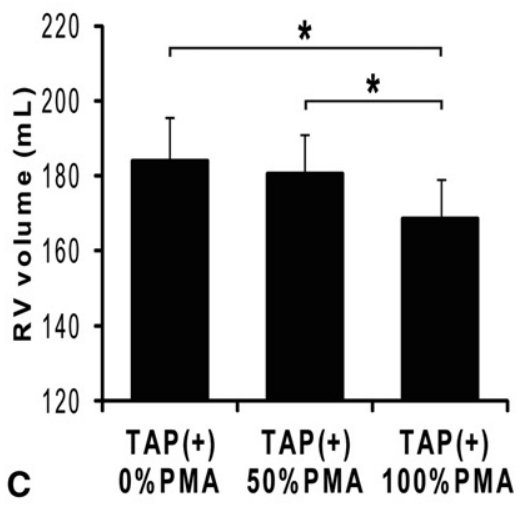

QA-M QA.S1 QA.S2 घA.P

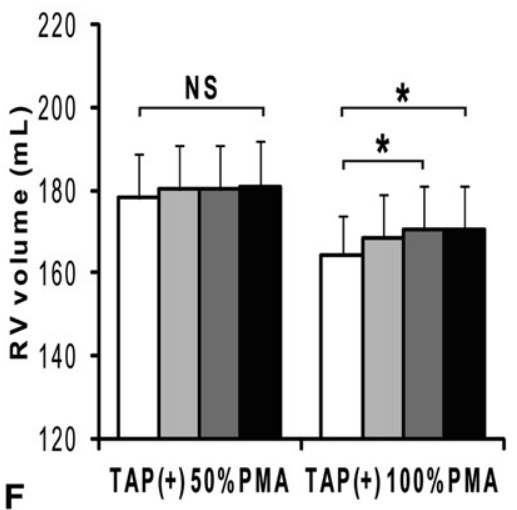

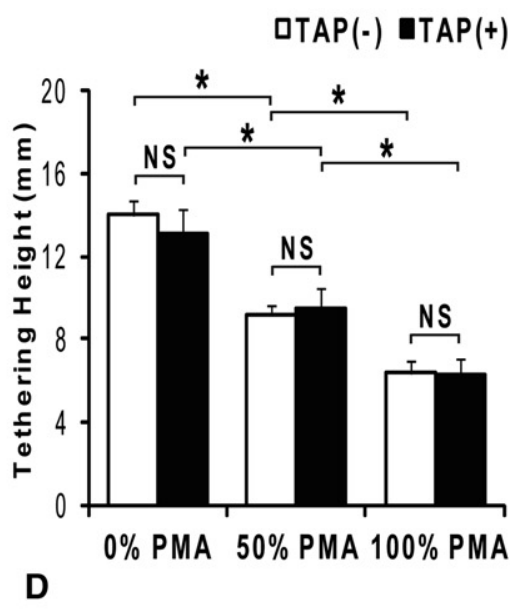

QA.M QA.S1 QA.S2 घA.P

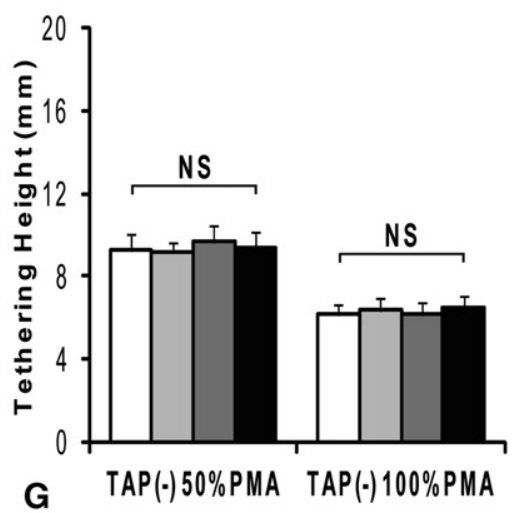

FIGURE 4. Changes in right ventricular $(R V)$ geometry (sphericity and volume) and tethering height by right ventricle-papillary muscle approximation $(P M A)$ and tricuspid annuloplasty $(T A P)$. A, The representative cross-sectional 2-dimensional echocardiographic images of the hearts in the groups without (-) annuloplasty $(0 \%, 50 \%$, and $100 \%$ papillary muscle approximation toward A-S1) and the group with (+) annuloplasty $(0 \%$ papillary muscle approximation), reproduced by the multiplanar 3-dimensional quantification mode of the Qlab software (Philips Healthcare, Andover, Mass). Arrows indicate the head of anterior papillary muscles. TV, Tricuspid valve. B, According to the 3-dimensional echocardiographic analysis, right ventricular sphericity index $(R V S I)$ was reduced by papillary muscle approximation toward the septum (A-S1, see Figure 1 for details of directions) regardless of annuloplasty. Right ventricular sphericity index was not significantly reduced by annuloplasty except in the $100 \%$ papillary muscle approximation group. NS, Not significant. Asterisk indicates $P<.05$ by least significant difference comparison test. C, Right ventricular volume was reduced by $100 \%$ papillary muscle approximation (A-S1). D, Tethering height of valve was reduced by papillary muscle approximation (A-S1) regardless of annuloplasty. Annuloplasty did not change tethering height. E and F, The A-M group showed the lowest right ventricular sphericity index and right ventricular volume among the papillary muscle approximation directions. G, There was no difference in the valve tethering height among the papillary muscle approximation directions according to analysis of variance. 
Among the RV-PMA directions, A-S1 (RV-PMA toward the midpoint between the medial PM and the posterior PM) reduced TR the most, although no statistically significant differences was found with the other directions (Figure 2, $B$ ). A-M (RV-PMA toward the medial PM) reduced RVSI the most (Figure 4,E), but it distorted the annulus (Figure $3, A$ ). In applying a more balanced force on the anterior PM in the anteroposterior direction, A-S1 or A-S2 (RV-PMA toward the midportion of the septum) may generate less stress on the PM than do the other directions. With respect to the height on the septum with which PM is approximated, we paid attention to keeping the PM sutures parallel to the tricuspid annulus to enhance tricuspid valve leaflet coaptation at the same height. Our preliminary trial, pulling the anterior PM obliquely toward the septal segment of the annulus, resulted in worsening of TR as a result of anterior leaflet prolapse (data not shown).

With respect to the extent of RV-PMA, the concept of this method started from the idea to restore all the valve structures (annulus, chordae, and PMs) closer to their normal position. It is unknown however, to what extent RV-PMA restores the PM to its "normal" position in this model. Given that $50 \%$ RV-PMA and TAP restore the $\mathrm{PM}$ and annulus, respectively, the group of $50 \%$ RV-PMA with TAP should demonstrate the "normal" condition of the tricuspid valve. Interestingly, 100\% RV-PMA worked better than 50\% RV-PMA to reduce TR in this study (Figure 2). Moreover, 100\% RV-PMA itself restored the tricuspid annular dimension similarly to the result seen with TAP (Figure 3, $B-D$ ) and also reduced right ventricular volume (Figure $4, C$ ).

Our ex vivo experimental setup allowed us to pass the sutures attached to the anterior PM of the right ventricle through the septum into the left ventricle (Figure 1, $B$ ). In an in vivo animal model or in a clinical setting, however, secure placement of the sutures to anchor the head of the anterior PM with septal wall in the dilated right ventricle is potentially challenging. We speculate that 2 or more pledget-reinforced mattress sutures would likely be required to distribute the forces applied and that pledget reinforcement of the sutures would be beneficial for a friable tissue.

\section{Study Limitations}

Porcine hearts were used in this study because the anatomic structures of the human heart and porcine hearts are similar with respect to the components of tricuspid valve (annulus, chordae, and PMs) and right ventricle. ${ }^{19}$ Our unique experimental model with a nonbeating flaccid porcine heart neither is physiologic nor mimics exactly functional TR. It does imitate the state of the arrested right ventricle after cardioplegia, however, and therefore does represent some of what the surgeon finds during passive saline solution testing. The intrinsically compliant tricuspid annulus and thin right ventricular wall enable intracardiac filling at relatively low pressure to expand the annulus and right ventricle while the valve closes, imitating the " $\mathrm{di}$ lated" tricuspid annulus and "spherical" right ventricle in systole, similar to the human mechanism of functional $\mathrm{TR}^{4}{ }^{4}$ The applied right ventricular pressures $(20-60 \mathrm{~mm}$ $\mathrm{Hg}$ ) are considerably higher than typical physiologic pressures, except in advanced disease states, which were mimicked by this model to distend the tricuspid annulus and right ventricle. Although it was not possible to keep the right ventricular pressure exactly constant while measuring TR flow, especially with massive TR, the effect of variable pressure was minimized. To confirm these proposed mechanisms, the results in this ex vivo model need to be validated in an in vivo model. In such experiments, one would need to assess the following questions: whether the TR reduction and the right ventricular geometric changes by RV-PMA affect cardiac stroke volume, or result in tricuspid stenosis, and whether the right ventricular remodeling effects of RV-PMA lead to a more competent tricuspid valve in the long term.

\section{CONCLUSIONS}

In summary, this ex vivo study suggests that RV-PMA potentially repairs TR better than TAP by improving not only annular dimension but also RVSI and valve tethering. Further investigations are necessary with in vivo animal studies to evaluate dynamic tricuspid valve function, right ventricular contractility, and durability of this technique.

\section{References}

1. Nath J, Foster E, Heidenreich PA. Impact of tricuspid regurgitation on long-term survival. J Am Coll Cardiol. 2004;43:405-9.

2. Cohn LH. Tricuspid regurgitation secondary to mitral valve disease: when and how to repair. J Card Surg. 1994;9(2 Suppl):237-41.

3. Navia JL, Nowicki ER, Blackstone EH, Brozzi NA, Nento DE, Atik FA, et al. Surgical management of secondary tricuspid valve regurgitation: annulus, commissure, or leaflet procedure? J Thorac Cardiovasc Surg. 2010;139:1473-82.e5.

4. Fukuda S, Gillinov AM, Song J, Daimon M, Kongsaerepong V, Thomas JD, et al. Echocardiographic insights into atrial and ventricular mechanisms of functional tricuspid regurgitation. Am Heart J. 2006;152:1208-14.

5. Fukuda S, Song J, Gillinov AM, McCarthy PM, Daimon M, Kongsaerepong V, et al. Tricuspid valve tethering predicts residual tricuspid regurgitation after tricuspid annuloplasty. Circulation. 2005;111:975-9.

6. Fukuda S, Gillinov AM, McCarthy PM, Stewart WJ, Song J, Kihara T, et al. Determinants of recurrent or residual functional tricuspid regurgitation after tricuspid annuloplasty. Circulation. 2006;114(1 Suppl):I582-7.

7. Spinner EM, Shannon P, Buice D, Jimenez JH, Veledar E, del Nido PJ, et al. In vitro characterization of the mechanisms responsible for functional tricuspid regurgitation. Circulation. 2011;124:920-9.

8. Calafiore AM, Gallina S, Di Mauro M, Gaeta F, Iacò AL, D'Alessandro S, et al. Mitral valve procedure in dilated cardiomyopathy: repair or replacement? Ann Thorac Surg. 2001;71:1146-53.

9. Kuwahara E, Otsuji Y, Iguro Y, Ueno T, Zhu F, Mizukami N, et al. Mechanism of recurrent/persistent ischemic/functional mitral regurgitation in the chronic phase after surgical annuloplasty: importance of augmented posterior leaflet tethering. Circulation. 2006;114(1 Suppl):I529-34.

10. Kron IL, Green GR, Cope JT. Surgical relocation of the posterior papillary muscle in chronic ischemic mitral regurgitation. Ann Thorac Surg. 2002;74 $600-1$. 
11. Langer F, Schäfers H. RING plus STRING: papillary muscle repositioning as an adjunctive repair technique for ischemic mitral regurgitation. J Thorac Cardiovasc Surg. 2007;133:247-9.

12. Matsui Y, Suto Y, Shimura S, Fukuda Y, Naito Y, Yasuda K, et al. Impact of papillary muscles approximation on the adequacy of mitral coaptation in functional mitral regurgitation due to dilated cardiomyopathy. Ann Thorac Cardiovasc Surg. 2005; 11:164-71.

13. Rama A, Praschker L, Barreda E, Gandjbakhch I. Papillary muscle approximation for functional ischemic mitral regurgitation. Ann Thorac Surg. 2007;84:2130-1.

14. Hvass U, Joudinaud T. The papillary muscle sling for ischemic mitral regurgitation. J Thorac Cardiovasc Surg. 2010;139:418-23.

15. Mandegar MH, Saidi B, Yousefnia MA, Alaeddini F, Roshanali F. Long-term effect of papillary muscle approximation combined with ventriculoplasty on left ventricle function in patients with ischemic cardiomyopathy and functional mitral regurgitation. Eur J Cardiothorac Surg. 2011;40:756-60.

16. Vasilyev NV, Padala M, Yamauchi H, Sill B, Delmo Walter EM, Thourani VH, et al. Functional and hemodynamic assessment of a novel 3-dimensional anatom- ically correct annuloplasty ring to correct functional tricuspid regurgitation. In: Proceedings of the 6th Biennial Meeting of the Society for Heart Valve Disease and Heart Valve Society of America; June 25-28, 2011; Barcelona, Spain. Available at: http://shvd.org/abstracts/2011/C8.cgi.

17. Bharucha T, Anderson RH, Lim ZS, Vettukattil JJ. Multiplanar review of threedimensional echocardiography gives new insights into the morphology of Ebstein's malformation. Cardiol Young. 2010;20:49-53.

18. Hoch M, Vasilyev NV, Soriano B, Gauvreau K, Marx GR. Variables influencing the accuracy of right ventricular volume assessment by real-time 3-dimensional echocardiography: an in vitro validation study. JAm Soc Echocardiogr. 2007;20: 456-61.

19. Crick SJ, Sheppard MN, Ho SY, Gebstein L, Anderson RH. Anatomy of the pig heart: comparisons with normal human cardiac structure. J Anat. 1998;193: 105-19.

20. Park Y, Song J, Lee E, Kim Y, Kang D, Song J. Geometric and hemodynamic determinants of functional tricuspid regurgitation: a real-time three-dimensional echocardiography study. Int J Cardiol. 2008;124:160-5. 